\title{
The Dominance of Deformation in Nuclei and Its Roots in a Symplectic Effective Field Theory
}

\author{
J.P. Draayer ${ }^{1}$, D. Kekejian ${ }^{1}$, G.H. Sargsyan ${ }^{1}$, T. Dytrych ${ }^{1,2}$, \\ K.D. Launey ${ }^{1}$ \\ ${ }^{1}$ Department of Physics and Astronomy, Louisiana State University, \\ Baton Rouge, LA 70803 USA \\ ${ }^{2}$ Nuclear Physics Institute of the Czech Academy of Sciences, \\ 25068 Řež, Czech Republic
}

Received 24 November 2021

doi: https://doi.org/10.55318/bgjp.2022.49.1.021

\begin{abstract}
To position in time our recent efforts to advance a forwardleaning many-particle shell-model theory for nuclear structure studies, we start with a review of major developments in nuclear physics over the last (20th) century, focusing especially on the last half as the mathematical framework that underpins our efforts was by-and-large developed by master subatomic theorists from the 50s through the 80s. Additionally, the landscape changed dramatically with the advent in the 90s of highperformance computing (HPC) facilities that could be used to test more complex theories that in prior times were deemed to be beyond reach. The first of these modern theories, the so-called no-core shell model (NCSM), taught us that one can carryout nuclear structure calculations using realistic interactions deduced from nucleon scattering data.

Subsequently we set out to explore whether this could be extended to algebraic models that involve non-compact group structures. A stretch goal of the latter campaign was to move away from a point-particlepicture of nuclei to a theory that is driven by the structure of the constituent nucleons themselves. What follows describes our efforts to date in moving towards this goal, and in the last section of this manuscript we proffered a novel symplectic effective field theory that may begin to pave the way for achieving this objective of breaking down barriers between a low-energy and high-energy view of nuclear physics, opening the door to what might best be called a truly unified ' $a b$ initio' theory for subatomic nuclear structure studies.
\end{abstract}

KEY WORDS: No-Core Shell Model (NCSM), Symplectic NCSM, SymmetryAdapted NCSM (SA-NCSM), Symplectic Effective Field Theory (Sp-EFT). 
J.P. Draayer, D. Kekejian, G.H. Sargsyan, T. Dytrych, K.D. Launey

\section{Introduction}

Subatomic physics is a relatively young science that in its earliest years was not only confronted with the wonders of a femto-scale world, but simultaneously with political events that ultimately helped ensure it would become an international discipline, one that today spans the globe with a smart and growing population of talented researchers that includes a 3rd and even 4th generation of scholars beyond what one might identify as the founding fathers of the field. Any attempt to identify by name all but a handful or so of these individuals would require far more space than that allotted for a contribution to this symposium, and of course it would be fraught with unintended omissions; nevertheless, given what is now unfolding, it seems appropriate to at least proffer some comments regarding the work of a few of the early contributors whose efforts helped to define focus areas within the discipline that to-date have stood the test of time.

Based on its history, the field of subatomic physics can be subdivided into two major focus areas advanced by research innovators. For subatomic physics the timeline spans the whole of the 20th Century, starting with the pioneering work of Ernest Rutherford [1] (England, Nobel Prize 1908) that ultimately led to the nuclear model of the atom that was advanced by his colleague Niels Bohr [2] (Denmark, Nobel Prize 1922), who in 1913 proffered his text-book view of the structure of the atom, and which ultimately led Bohr to focus his attention on nuclear physics starting with a relatively simple liquid-drop picture of nuclei, that supported vibrational as well as rotational modes and under the right conditions even nuclear fission. This was followed by two game-changing developments, both products of the late $40 \mathrm{~s}$ and early $50 \mathrm{~s}$; specifically, work that built forward from that of Niels Bohr by his son Aage Bohr and Ben Mottelson (Copenhagen School, Nobel Prize 1975, shared with Rainwater), generally known as a collective model of nuclear structure [3], and another based on the pioneering work of Maria Goeppert-Mayer and J. Hans D. Jensen [4] (Nobel Prize 1963, shared with Eugene Wigner) for advancing a particle-based shell-model picture of nuclei, which in its simplest form amounts to placing neutrons and protons into a three-dimensional harmonic oscillator (3D-HO) potential with a spin-orbit term added to account for the separation of levels within a given shell.

The evolution towards modern views of nuclear structure proceeded along two lines of discovery: the quantization of a liquid-drop that can rotate and vibrate - the Rotation-Vibration Model (RVM) that grew into the General Collective Model [5] (GCM; German/Frankfurt School led by Walter Greiner in the early 60s) along with various associated mean-field efforts; and, attempts to expand the single-particle picture into a many-particle shell model theory that respects the fermion character of its constituent (neutron and proton) parts. While the former required a quantization of a 'nuclear fluid', the latter turned on mathematical developments as there was a need for a discrete-particle picture that gave rise to the collective modes that were an integral part of the GCM. 
The latter led in 1958 to the development of the so-called SU(3) shell-model [6] (British/Sussex School led by J. Phil Elliott), which showed that the valence space of a many-particle, harmonic-oscillator-based shell-model theory could be reorganized into irreducible representations (irreps) of SU(3), where the latter with its two (Casimir) invariants, one of 2 nd order and another of 3rd order could be directly linked to the $\beta$ (prolate) and $\gamma$ (triaxial) features of the collective model. The latter grew into what today is a major enterprise called an algebraic approach to nuclear structure, as it relied on a group-theoretical framework with subgroup chains that are related to specific physical phenomena.

Because of the beauty and complexity of the latter, there have been many efforts to develop simpler realizations of this structure that could be used to circumvent the complicating antisymmetry requirements of a fermion-based theory. The best illustration of this is captured in the so-called Interacting Boson Model [7] (IBM, with Franco Iachello in the lead) which has itself developed into a major enterprise that continues even until today to dominate our field. While the basic assumptions of the IBM are an oversimplification, it is perhaps the best algebraic theory available for gaining a quick-and-simple, text-book-like understanding of nuclear structure - not unlike what the Bohr Model did for atomic physics. The basic tenants of the theory depend upon an assumption that the primary modes found in nuclei can be represented in terms of ' $\mathrm{s}$ ' and ' $\mathrm{d}$ ' bosons that respectively carry angular momentum 0 and 2 , with bilinear products of these six modes defining the generators of a U(6) symmetry group that has three subgroups chains that track, respectively with rotations in its SU(3) limit, vibrations in its $\mathrm{U}(5)$ limit, and $\mathrm{O}(6)$ in the case of triaxial shapes.

It is against this background that we now turn our attention to the development of so-called no-core shell-model (NCSM) theories, which are fully microscopic in their management of the many-particle dynamics. While much of the algebraic ground work associated with these 'no-core' approaches came in the 70s and $80 \mathrm{~s}$, the usefulness of these efforts was quite limited as they could only be appreciated through various phenomenological limits similar in spirit to what is used in the IBM, because the combinatorial growth in the dimensionality of the model spaces that one encounters when attempting to take into account - for example, a mixing of giant monopole and quadrupole modes into this picture, a feature that is required to be able to reproduce measured $B(E 2)$ strengths even within simple valence spaces - quickly exceeds that which can be accounted for by incorporating even a few valence shells without introducing a compromising assumption into this picture called an effective charge. (The inclusion of a single additional shell more than doubles the sum total of all previous basis states - that is,the model spaces grow combinatorially with increasing nucleon numbers and additional shells that they may occupy.)

The latter is and remains the Achilles' Heel of any realistic many-particle shell model theory. But, this picture began to change in the 90s with the advent of high-performance computing (HPC) facilities that for the first time could be 
used to test the real value of a no-core shell-model (NCSM) theory that accommodates ' $a b$ initio' interactions and is robust enough to allow for configuration mixing across single-shell boundaries. But even with access to such HPC environments, the combinatorial growth in the size of the model spaces needed to gain convergence to important experimentally measured quantities fell short, even when huge allocations of run times to carry out calculations on the biggest and best of these systems were made available.

The rest of this manuscript is devoted to the 'rest of the story'; namely, building upon lessons learned from NCSM outcomes, to what today flies under the banner of a symmetry-adapted no-core shell model (SA-NCSM) theory [8-11], one that respects the microscopic nature of the NCSM while managing the 'explosive growth' in the size of the model spaces through natural (symplectic) subspaces that can: 1) be ordered and trimmed in terms of their relative importance to ensure maximum reach of the theory, and 2) be partitioned into semi-independent blocks to capitalize on parallel processing technologies to reproduce - both without a need to introduce effective charges to reproduce observed enhanced B(E2) rates, which is 'far-and-away' the single most important feature in nuclei. In short, the SA-NCSM is aligned with the 'dominance of deformation' that is pervasive across the chart of the nuclides.

\section{Transitioning from a Single-Shell to a Multi-Shell Environment}

The NCSM, named and advanced by Peter Navrátil, James Vary and Bruce Barrett [12], is a complete many-particle shell-model theory that includes all multi-particle, multi-hole configurations up to some $N_{\max }$ cutoff that designates a maximum number of harmonic oscillator excitations that are allowed beyond those of the lowest ground-state configuration $N_{\max }=0$ ). The NCSM designation is often qualified with an 'ab initio' prefix to stress that in applications of the theory realistic interactions fit to nucleon-nucleon scattering data are used. It satisfies all the antisymmetrization requirements of such a system as it uses a Slater determinant set of basis states build up from single-particle states. Since its inception, the NCSM has served as the fully-microscopic shell-model theory of choice for determining the structure of many-particle nuclear systems.

Having learned of the NCSM from its authors at an American Physical Society meeting held in Washington DC in the early 2000s, and having delved into similar matters myself in the 80s and the $90 \mathrm{~s}$ - albeit from an algebraic perspective and with a number of students and colleagues [13-16] - I discussed the possibility of creating a symmetry-adapted version of the NCSM with members of my LSU-based graduate student team which at that time included - Kristina (Sviratcheva) Launey from Bulgaria and Tomáš Dytrych from the Czech Republic. My optimism that a symmetry-adapted version of the NCSM could be created, was based on my prior work in this arena, dating back to my thesis on 'A Deformed Potential Many-Particle Theory' plus postdoctoral stints at the Niels 
Bohr Institute in Copenhagen (1968-69), the University of Michigan (1970-73 with Ted Hecht), and the University of Rochester with Bruce French (1974-75) along with many-others - including, especially interactions with David Rowe and George Rosensteel (more below). And I was also quite certain that if we were to be successful with such an undertaking that we would also be able to beat back the explosive growth issue through the use of cleverly chosen basis states which would ensure convergence of results with respect to the issue of enhanced B(E2) transition rates, without a need for introducing effective charges into the theory.

Nevertheless, this came with a recognition that such a campaign might take a dozen years or so to unfold, which we broke out into three four-year parts with the assumption of a go-nogo decision being taken every 3rd or 4th year on whether to continue or not and/or make mid-course adjustments based on what we had learned up to each checkpoint. Despite the risks, this small group chose to move forward together! One could challenge such a decision - and it was, especially by my fellow faculty members - as it was a high-risk plan, especially with graduate student involvement as this meant that early in their career they would be committing to a graduation time line that could be about double normal expectations; nevertheless, a decision was made to move forward, and we did. I believe that in this case the end has more than justified the means! However, in hindsight, I would add that doing something like this once in one's career is probably more that enough! Kudos to Kristina (Bulgaria) and Tomáš (Czech Republic) for all they did and are continuing to do to advance this ambitious agenda; it would not have happened without their support! Also, as reflected in this leadership team, international collaboration has been and continues to be a main-stay consideration in our commitment to advancing the SA-NCSM agenda, which continues to expand in various new and interesting ways!

\section{The Symmetry-Adapted No-Core Shell Model (SA-NCSM)}

Moving from a single-shell theory to an open multi-shell theory of the type encountered in NCSM applications can be viewed in two ways: The first is what I will refer to here as a 'particle picture' as it tracks with a 'point-particle-picture' of the nucleus referenced earlier, while I will refer to the second as an 'energy picture', which we will pick up on after finishing a more thorough description of the 'particle picture'. Suffice it to say for now that the 'particle' versus 'energy' pictures proferred here also anticipates the last section of this manuscript where the concept of a symplectic effective field theory for understanding nuclear structure is introduced. And to further stress the importance of such a notion in studying the structure of nuclei, it seems useful to recall the importance as well as the uncertainty associated with the 'wave-particle' duality concept in quantum mechanics!

The simplest 'particle' picture for nuclei tracks back to the earliest work on 
the development of the shell-model itself. Its usefulness in describing an open multi-shell environment of the type encountered in a NCSM theory would seem to be straightforward. For example, consider the effect of lifting a single nucleon out of its valence shell by giving it an energy boost of $2 \hbar \omega$. (The parity of the harmonic oscillator levels alternate, and hence the factor of 2 that multiplies the $\hbar \omega$ is required to ensures parity conservation.) Historically this has been called a $1 \mathrm{p}-1 \mathrm{~h}, 2 \hbar \omega$ excitation of the system. But in fact, even from this simplest of all perspectives one can see that such a picture is an oversimplification because of the antisymmetrization requirements associated with satisfying the Pauli Principle require that an energy boost of this type ( $2 \hbar \omega$ excitation) has to be shared with any and all other identical (indistinguishable) nucleons in a similar way. Specifically to this point, the latter must allow for the possibility of such a boost in energy engaging $2 \mathrm{p}-2 \mathrm{~h}$ excitations with each member of the pair gaining a $1 \hbar \omega$ boost, and so on. Similarly, when a $4 \hbar \omega$ boost is added to the system it is clear that the Pauli allowed excitations begin to mushroom in their complexity, into for example, one particle up $3 \hbar \omega$ and another by $1 \hbar \omega$ or two pairs excited by $2 \hbar \omega$ each, and so on.

From rudimentary considerations of this type one can deduce that the number of particle-hole excitations for any (even parity) allowed energy boost outstrips the ability of a simple 'particle' picture to manage and track its growth in a simplified fashion. The NCSM handles this dilemma through the use of a set of Slater determinants in the single-particle basis states. But with the latter comes other challenges, such as how to best ensure that calculated eigen-solutions do not contain spurious center-of-mass excitations. But all-in-all, it seems there are relatively simple ways to manage most if not all such issues, without compromising the need to ensure that all physically relevant configurations - and no more - are appropriately taken into account, even if the onus for ensuring this is relegated to the Hamiltonian itself, with 'simplicities within complexity' of such solutions having to be recognized in advanced and projected out of calculated eigen-solutions to test their relevance, which would amount to identifying a new basis that exposes these features in advance, which brings us back full circle to the notion and need for a SA-NCSM, and in particular, to the genius of the Elliott SU(3) Model in providing a path forward.

\subsection{From the Elliott's SU(3) model to the symplectic picture}

The Elliott SU(3) Model is an excellent example of a 'particle' picture of nuclei, and one that seems to be ready-made for adoption into an open multi-shell environment, especially because SU(3) is the symmetry group of a single valence shell in nuclear physics. But before jumping into such a discussion, it is important to appreciate the role the Elliott SU(3) Model plays in helping to bridge the gap between the use of a collective model picture for nuclear physics and the many-particle shell model picture. 
The genius of Elliott's SU(3) picture lies in the fact that one can reorganize the many-particle basis basis states of single 3D-HO harmonic oscillator shell into irreducible representations (irreps) of SU(3), where each irrep, labeled by $(\lambda, \mu)$, can in turn be associated with the corresponding $(\beta, \gamma)$ shape variables of the geometrical collective model. This can be achieved by aligning invariant measures of the two theories; specifically, the second-order invariant of SU(3), $C_{2}(\lambda, \mu)$, is proportional to $\beta^{2}$, while the third-order invariant, $C_{3}(\lambda, \mu)$, is linked to $\beta^{3} \cos (3 \gamma)$, and so on. As will be discussed in greater detail below, this Elliott breakout into $\mathrm{SU}(3)$ states for any distribution of particles in any shell of the 3D-HO, extends to the whole of the 3D-HO.

Having laid down this groundwork, we now turn to a fuller discussion of the 'energy' picture, as an attractive alternative to the 'particle' picture, a shift that is fascinating for its mathematical beauty as well as its physical simplicity. In short, when applied, it can be used to help with the factorization of the problem into two parts, the first focused on a "core" (particle) part that is responsible for addressing the antisymmetrization requirements of the Pauli Principle, and a second 'cloud' (energy) part that accounts for the collective (particle-neutral) modes that support enhanced B(E2) transition rates. The best way to gain a deeper appreciation for this picture is to understand at a deeper level ramifications of the fact that the $\operatorname{Sp}(3, \mathrm{R})$ group (non-compact with infinite dimensional irreps and 21 generators) is the dynamical symmetry group of the $3 \mathrm{D}-\mathrm{HO}$ as a whole, which means one must track the shell of origin of particle clusters and subclusters, including specifically their SU(3) character, and top each of these off with collective $\mathrm{Sp}(3, \mathrm{R})$ boson-like excitations [17].

\subsection{Embedding the symplectic scheme into the SA-NCSM}

From a structure perspective, the 21 generators of $\operatorname{Sp}(3, \mathrm{R})$ span the space of all independent operators that are quadratic in the system's momentum and coordinate variables. These can be rearranged into $62 \hbar \omega$ raising ( $\uparrow$ ) operators with SU(3) tensor character $(2,0)$ and the conjugate $62 \hbar \omega$ lowering $(\downarrow)$ operators with $\mathrm{SU}(3)$ tensor character $(0,2)$, plus 9 other $0 \hbar \omega$ horizontal $(\leftrightarrow)$ operators that, respectfully are generators of the compact (finite-dimensional) U(3) subalgebra of $\mathrm{Sp}(3, \mathrm{R})$ that can be further reduced into the eight generators of $\mathrm{SU}(3)$, with $\mathrm{SU}(3)$ tensor character $(1,1)$, and another single operator with SU(3) tensor character $(0,0)$ that is an operator that counts the total number of oscillator quanta at any level within the symplectic irrep.

It should be clear from this picture that one can create a 'symplectic cloud' by climbing up the symplectic ladder, starting from the bottom-most rung, called the lowest-weight state of the irrep, which means that an application of any of the $62 \hbar \omega$ lowering $(\downarrow)$ operators acting on the state annihilates it. And further, that through multiple applications of the $62 \hbar \omega$ raising $(\uparrow)$ operators as well as the 9 other in-shell $0 \hbar \omega$ horizontal $(\leftrightarrow)$ operators to the lowest-weight state 
one can span the space of all states within that symplectic irrep. And further, when this formula is applied to the 3D-HO vacuum, $|(0,0)\rangle$ (no particles in the oscillator), where in this expression $(0,0)$ literally means $(\lambda, \mu)=(0,0)$ since the starting state in this case (see below, also called the 'bandhead configuration' of the corresponding symplectic irrep) is that of an empty 3D-HO.

It should also be clear from the above, that the $(\lambda, \mu)=(0,0)$ symplectic superstructure is a special particle-neutral vertical extension that spans the whole of the infinite-dimensional (non-compact) representation that can be coupled to any particle distribution or distribution(s) that is(are) free of any/all such symplectic excitations. In other words, this particular construction, which could be called any one of a number of things, but our previous discussion regarding 'particle picture' versus 'energy picture' it is in reality a 'virtual energy cloud' consisting of all possible small (within a shell) and big (between neighboring shells) of monopole and quadrupole types that can be used to capture and represent the internal dynamics within a NCSM picture of nuclei in an organized manner.

This separation of modes, particle versus energy, is what the SA-NCSM exploits. The biggest challenge is dealing with what is left behind in the 'particle sector', as there is no need to worry about how best to fold in the $\mathrm{B}(\mathrm{E} 2)$ enhancing modes, but this too can be simplified, as it breaks up into a 'sum of clusters' that exploits a generalization of the Elliott SU(3) scheme that itself can be systematically organized into $\mathrm{SU}(3)$ bandhead configurations, free of the collective modes of the symplectic type, that can be built up for any $A(Z, N)$ nucleus drawn from a collection of subsystems, $A_{i}^{\left(\lambda_{i}, \mu_{i}\right)}\left[Z_{i}^{\left(\lambda_{i}, \mu_{i}\right)}, N_{i}^{\left(\lambda_{i}, \mu_{i}\right)}\right]$, where the sum of the $A_{i}$ 's, $Z_{i}$ 's, and $N_{i}$ 's pieces is, respectively $A, Z$ and $N$. The SA-NCSM handles all of this, and the vertical extension of each cluster or subcluster, as may be necessary, that can be expanded or contracted individually depending on their relative importance to the whole, etc. We now turn to a few applications of the SA-NCSM, to demonstrate the effectiveness of this scheme in generating converged results that are of practical interest.

So it seems a picture that emerges is a relatively simple one: Any Pauli allowed valence distribution of $A$ nucleons ( $Z$ protons and $N$ neutrons) has a set of SU(3) $(\lambda, \mu)$ irrep values associated with it, each of which may occur multiple times but with each reflecting a unique internal structure of independent SU(3) bandhead configurations - all of Elliott type - to which one can couple via a direct product of multiple $2 \bar{\omega}$ excitations; that is, $(\lambda, \mu) \times[(2,0) \times(2,0) \times \ldots]$, out to some $N_{i}^{\left(\lambda_{i}, \mu_{i}\right)}$ factors of $(2,0)$ where in this case, unlike what is done in the case of the NCSM, the latter can be tied to the $(\lambda, \mu)$ value of the bandhead itself. Pushing this analogy further out, the $(\lambda, \mu) \times[(2,0) \times(2,0) \times \ldots]$ product can be interpreted as a swarm of $\mathrm{S}$ and $\mathrm{D}$ bosons being added to the bandhead, where the latter serves to mimic couplings to the giant monopole and giant quadrupole modes which in turn serve to account for the observed enhanced B(E2) rates among the member of low-lying bands. And pushing even harder, it should also be clear that such a 'boson cloud' itself has the same algebraic structure as that 


\section{Dominance of Deformation in Nuclei and Its Roots in a Sp-EFT}

associated with IBM model and therefore should itself display the U(6) structure of the IBM with its three (rotational,vibrational, or gamma soft) subgroup features.

\subsection{Towards a field theory view of the symplectic picture}

In anticipation of the last section of this manuscript, it is important to understand this 'energy' picture from a more geometrical perspective. For this I pose the following question: 'How can it be that the Hoyle state in ${ }^{12} \mathrm{C}$, alleged to be a $4 \mathrm{p}-4 \mathrm{~h}$ configuration in a 'particle' picture of things, enjoys a $4 \hbar \omega$ boost in energy above the ground state that magically becomes the next-up configuration, falling below a $2 \hbar \omega 2 \mathrm{p}-2 \mathrm{~h}$ state?' It would seem odd to think that the constituent particles ( 6 protons and 6 neutrons) give up some of their own self-energy to enable the system as a whole to explore its surroundings (phase space) in an attempt to find an alternative preferred arrangement without violating overall energy conservation! Might a better answer be that there is a virtual 'cloud of (massless) bosons' associated with the oscillator itself that serves as an enabling agent that allows the $A(6,6){ }^{12} \mathrm{C}$ system to explore alternative configurations and in so doing finds a preferred substructure - such as a special arrangement of 3 tightly bound alpha-particle-like clusters in the case of ${ }^{12} \mathrm{C}$, that in the aggregate is more tightly bound when allowed to self-arrange into a geometrically favored configuration that lies lower energetically than that of a $2 \mathrm{p}-2 \mathrm{~h}$ state - or any other configuration - but which necessarily must lie above the ground state? It seems the latter is a far simpler and better picture of the dynamics that is really in play. So, in summary, it seems that a simple 'collection of all possible Pauli allowed 'bandhead configurations' plus a 'cloud of massless S and D bosons' that can attach itself to any such bandhead may be the simplest and the most compelling way to view the structure of atomic nuclei. As shown ahead in the final sections of this manuscript, this picture is aligned with the concept of an 'effective' symplectic field theory for nuclear structure studies.

The picture painted above suggests that the SA-NCSM is a collection of $\operatorname{Sp}(3, \mathrm{R})$ defined symplectic towers built on all the allowed SU(3) configurations that are free of collective $(2,0) \mathrm{SU}(3)$ substructures; stated otherwise, the $(0,2)$ lowering operator of the $\operatorname{Sp}(3 . \mathrm{R})$ algebra acting on any bandhead configuration destroys it. This, in turn, puts the onus on one being able to construct all the Elliott SU(3) bandheads for a particular $A(Z, N)$ nucleus, where this set depends upon the number of shells included in the analysis and the distributions of particles among those shells. While not trivial, this can be done because there exists a simple algorithm that gives all possible $\mathrm{SU}(3)$ configurations for every distribution at every level, starting at the bottom $A(Z, N)=0(0,0)$ level (no nucleons) and moving upward to $A(Z, N)=1(1,0)$ (proton) and $1(0,1)$ (neutron) to $2(2,0)$ (di-proton), 2(1,1) (deuteron), 2(0,2) (di-neutron), and so on. The SA-NCSM basis generator subpackage of the SA-NCSM code does this for any $A(Z, N)$ nucleus and the associated distributions. 


\section{Results that Illustrate the Effectiveness of the SA-NCSM}

So, while it may not be overly difficult to expand the set of bases states in this way, it has to be done carefully and correctly so as to not unintentionally exclude important new bandhead configurations that could well be associated with new and distinct physical modes every step of the way. As an important example, consider the pairing modes that are known to break symplectic symmetry and be physically important even to the point of challenging the dominant collective features that are carried forward through the quadrupole coupling terms. These must be a part of the set of all other bandheads; not including these can lead to a picture that misses some important physical phenomena and various other inconsistencies. The good news is that the SA-NCSM as it currently exists takes account of all of these modes, so one does not need to understand the details of the code as to its completeness of the basis states being used because this has been build into and thoroughly tested using various dimensionality sumrule checks. In what follows we will see the importance of this in results for ${ }^{12} \mathrm{C}$, which show a first set of results that ignores irrep mixing caused by the singleparticle spin-orbit interaction, and a second set of results with it turned on.

\subsection{Early results for ${ }^{12} \mathrm{C}$ [with \& without spin-orbit $(l \cdot s)$ splitting]}

The results shown in Figure 1 are from one of our early studies in which we used a version of the so-called NCSpM $[18,19]$, which was then and still remains a good predictor of SA-NCSM results, to study the structure of ${ }^{12} \mathrm{C}$.

The goal was to see if we could gain better insight into the nature of the Hoyle state that has attracted the attention of many researchers over several decades because ${ }^{12} \mathrm{C}$ was then and still is known to be the 'main element that forms all structures of life'. When the NCSM was applied to ${ }^{12} \mathrm{C}$, the results were inconclusive because even when pushed out to $N_{\max }=10$, which is the best that could be done at that time, the theory showed very little evidence of convergence. (Once the SA-NCSM was up and running, our results for ${ }^{12} \mathrm{C}$ were tested against those for NCSM at the $N_{\max }=8$ level - which was the best we could do at that time - to ensure that our results agreed with the NCSM, and so on.) But from that exercise we were in fact able to identify the particle-hole character of the states that appeared to be diving down towards becoming the Hoyle state and another near-lying state, which we now believe is the $2 \mathrm{p}-2 \mathrm{~h}$ configuration. The $\mathrm{p}-\mathrm{h}$ labels in the Figure 1 (upper panel, lefthand side) tell this story.

But there was more to be learned from the results for the ${ }^{12} \mathrm{C}$ case. Specifically, the results clearly show the dominance of a single symplectic irrep within each of the states that were found to be diving strongly downward, with each using up nearly the full strength of the eigenstates and therefore the series for each could be extended by adding only maximally deformed and stretched SU(3) configurations at every level of the tower; namely, keeping only maximally de- 

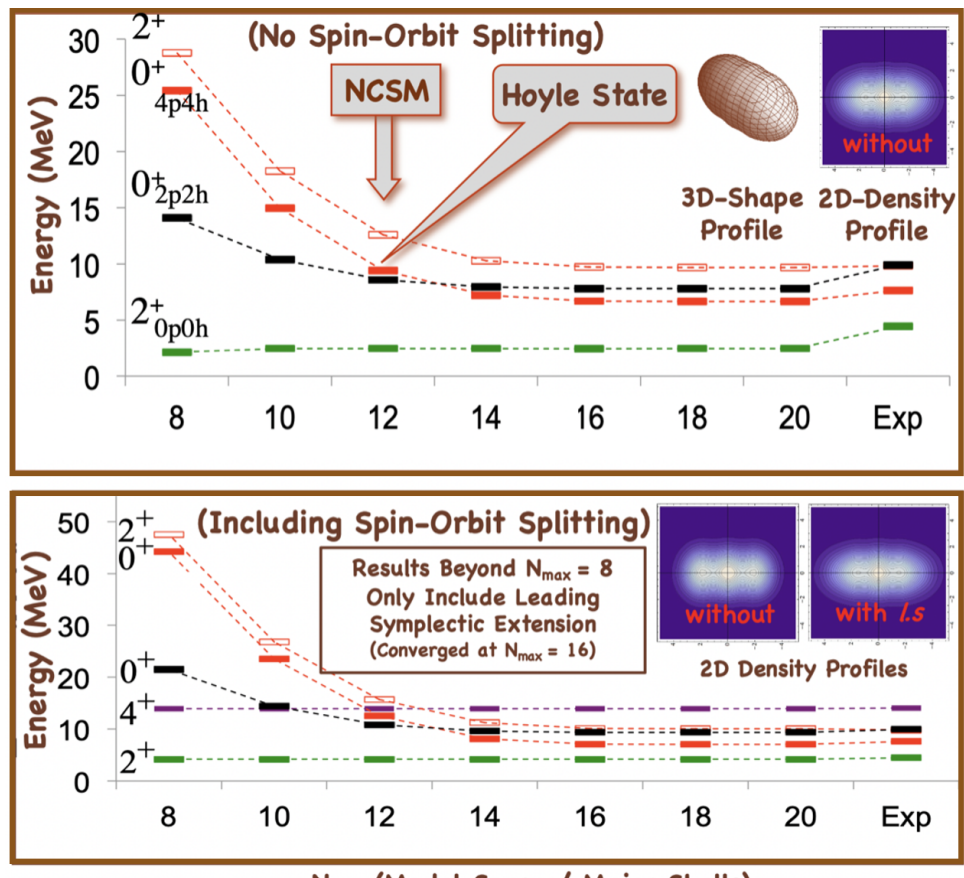

$\mathrm{N}_{\max }$ (Model Space / Major Shells)

Figure 1. (Color online) SA-NCSM results for ${ }^{12} \mathrm{C}$, without (upper panel) and with (lower panel) spin-orbit induced mixing at bandhead level. Convergence for Hoyle state is achieve at $16 \hbar \omega$.

formed irreps as one assends up and along the most deformed edge of the tower: $(\lambda+2, \mu),(\lambda+4, \mu), \ldots$ and so on. This feature is reflected in both inserts, with full convergence being met at $N_{\max }=16$. (We extend this exercise out to $N_{\max }=20$ to show that convergence had been met, and held steady all the way out to $N_{\max }=20$.)

And further, we also knew that the SU(3) character of the valence shell structure is challenged by the single-particle spin-orbit interaction $(l \cdot s)$ because it is a symplectic symmetry breaking interaction that is probably second only to the primary quadrupole interaction so we added it - and only it - to the Hamiltonian and only at the ground-state (bandhead) level; and, consistent with keeping maximally deformed irreps as one moves up along the edge of the symplectic ladder, letting its effect propagate upward via the changes that it induced at the bandhead level. Much to our surprise and delight, the addition of this symplectic breaking term to the bandhead picture improved matters, as can be seen from the results shown in the second (lower) panel; specifically, it served to bring the calculated results in clearly better alignment with experiment. What is not shown, but also the case, the latter yields correct B(E2) transitions rates among all the 
low-lying states of the system and the rms system radii that are spot on with the experimentally measured numbers. In Figure 1 we have also included on both subpanels some further artwork (a 3D-Shape Profile in the first panel, along with a 2D-Density Profile); and two 2D-Density profiles in the lower panel, that seem to further affirm that the structure of ${ }^{12} \mathrm{C}$ is now understood at a much deeper level than previously deemed possible.

\subsection{Recent results for ${ }^{48} \mathrm{Ti}$ [neutrinoless $(\beta \beta)$ beta decay]}

To show some recent results associated with the use of the SA-NCSM [20], see Figure 2, where we display some results for ${ }^{48} \mathrm{Ti}$ that are of interest in studies of the nature of neutrinos, finding if a neutrino is (or is not) its own anti-particle through, for example, studies of neutrinoless double- $\beta$ decay, and thereby affirming or challenging the Standard Model in particle physics that has to date stood the test of time in dealing with the nature of the strong interaction, but less so regarding the nature of the weak interaction.

This is part of the thesis work of Grigor Sargsyan [21] (LSU Spring 2021), who is a classmate of David Kekejian, both from Armenia - who expects to graduate this semester (LSU Fall 2021). Details behind the theory, which are not given here, should be available in the Spring 2022. What this Figure 2 does show is that the SA-NCSM can be used to address important questions about heavier nuclei, and that the dimensionality savings it can bring forward relative to that

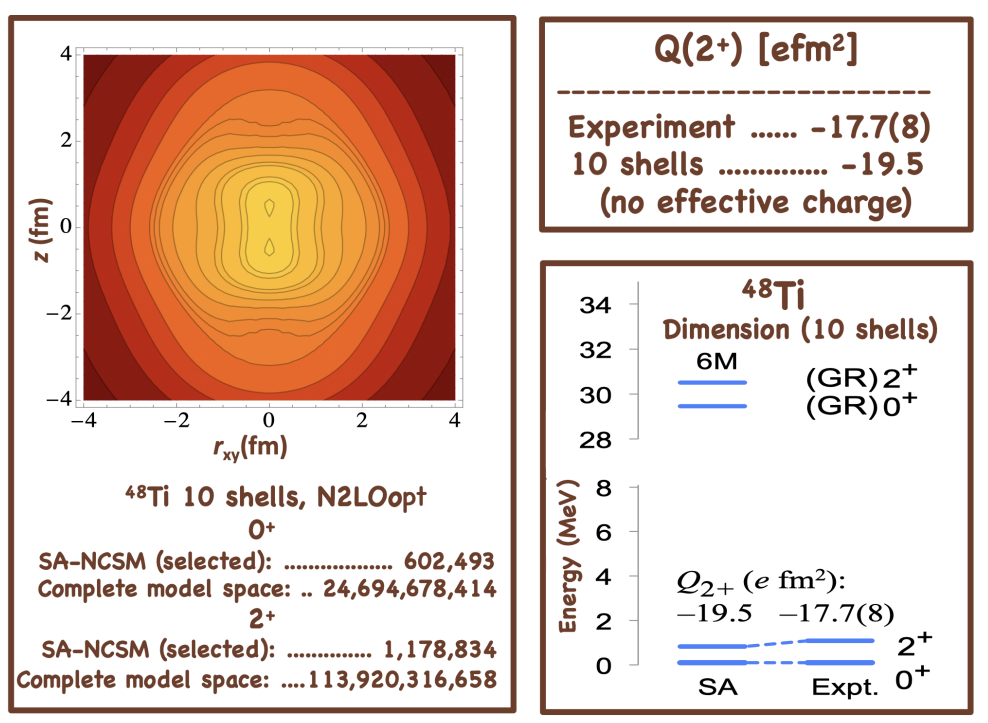

Figure 2. (Color online) Result for ${ }^{48} \mathrm{Ti}$, which is of interest in studies of neutrinoless $(\beta \beta)$ beta decay. 
of the NCSM are staggering! Also note that in this case, as in others we have explored, at even an 8-shell cutoff level the known $\mathrm{Q}\left(2^{+}\right)$values are aligned with experiment.

\section{Proposed Symplectic Effective Field Theory (Sp-EFT)}

Showing that the 'dominance of deformation' claim for nuclei has it roots in a symplectic effective field theory (SpEFT) is the thesis project of David Kekejian [22]. The thrust of his research program is exploring the origins of symplectic symmetry from a simple real scalar field theory Lagrangian with two parameters that set the scale of the relevant physics and the strength of the interaction. It is a four-dimensional dynamical theory that explains many features of nuclei such as rotations and vibrations, the observed enhanced $\mathrm{BE}(2)$ values and radii of nuclei.

The SpEFT concept explains the 'symplectic cloud' picture proffered in subsection 3.2 through the fields introduced within a Lagrangian formalism, which in turn suggests that the SA-NCSM is really the aggregate of multiple symplectic NCSpM towers built on a complete set of all independent many-particle configurations of an $A(Z, N)$ nucleus where the latter is organized according into all independent SU(3) bandheads of the Elliott type up to some $N_{\max }^{(\lambda, \mu)}$ cutoff for each. The latter set must be complete and satisfy the antisymmetry requirements of the Pauli Principle within and among all spurious-free $(\lambda, \mu)$ bandhead configurations included in a particular application of the theory, to which is added as a direct product a 'cloud of (S and D) bosons'. While details await publication of David Kekejian's thesis, the underpinning of the theory is captured in Figure 3.

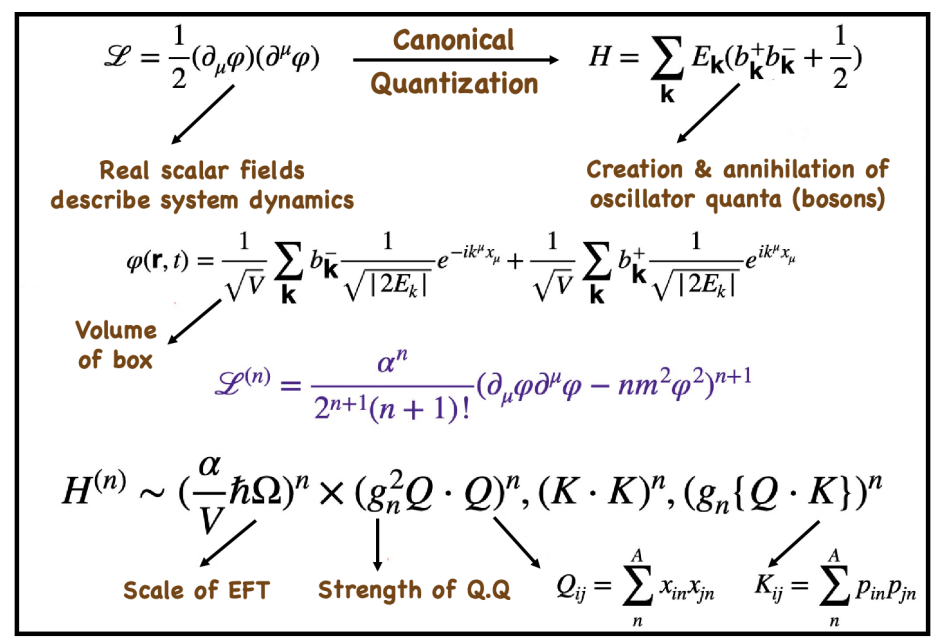

Figure 3. (Color online) A schematic for constructing an EFT for nuclear structure studies. 
J.P. Draayer, D. Kekejian, G.H. Sargsyan, T. Dytrych, K.D. Launey

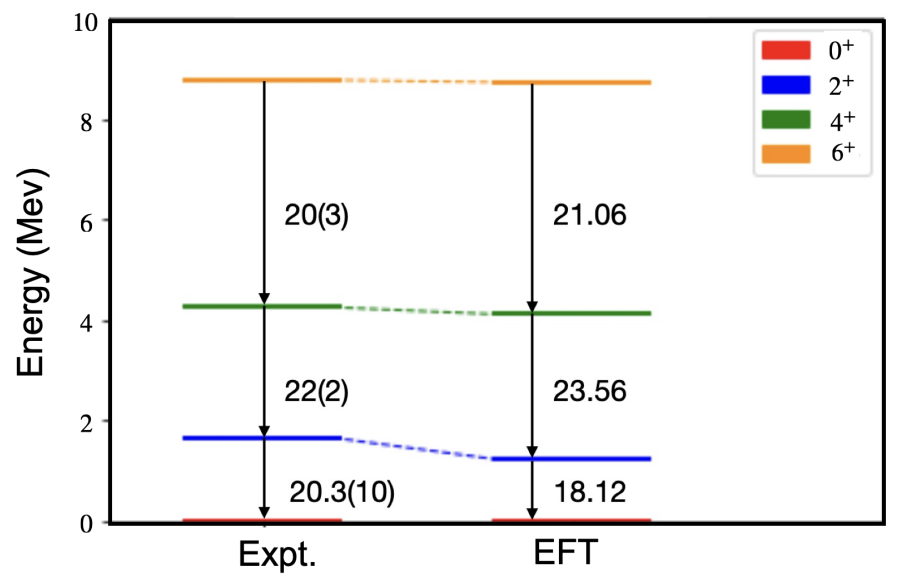

Figure 4. (Color online) Results of an application of the SpEFT theory to ${ }^{20} \mathrm{Ne}$.

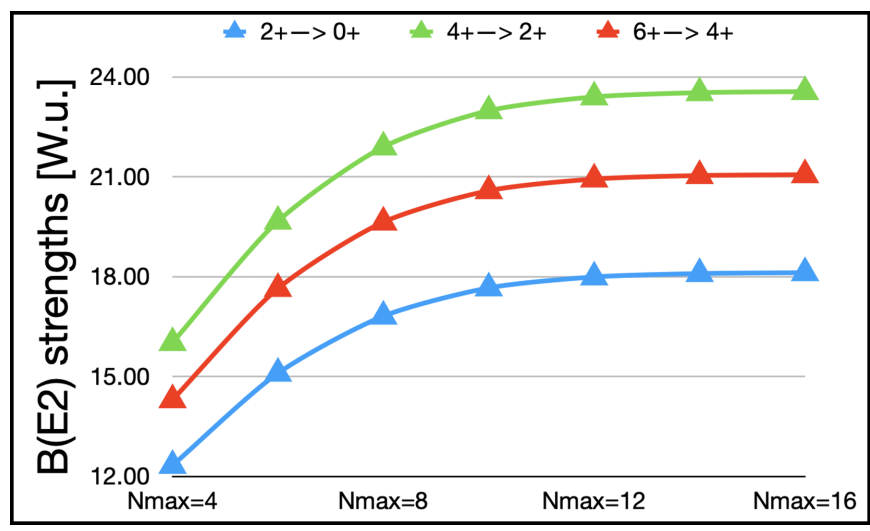

Figure 5. (Color online) Results of an application of the SpEFT theory to ${ }^{20} \mathrm{Ne}$.

Note the convergence to the experimental results (spectrum and B(E2) transition rates) as a function of $N_{\max }$. And also note the convergence to experimental B(E2) values is reached at $N_{\max }=16$, which is the same as for the ${ }^{12} \mathrm{C}$ case discussed in subsection 4.1. And the convergence at lesser $N_{\max }$ values drops off smoothly, but precipitously and though not illustrated do not follow that of a rigid rotor dynamics.

\section{Conclusion}

The story of a campaign to create a fully microscopic, symmetry-adapted nocore shell-model (SA-NCSM) for nuclear structure studies is told, and to place 
it in a correct historical context, starting from the beginning of the last century, while presenting in somewhat greater detail the storyline of its evolution through the last half of that century, and how the high-performance computing (HPC) revolution of the 90s intersected with this in a way that has lead to a demonstration that could help it become the new theory of choice for carrying out future deeper dives into the structure of atomic nuclei, beyond than even thought possible just prior to the close of that century.

The story is one of rolling progress into the current century that provides everclearer evidence that there is 'simplicity within complexity' in nuclear physics. And it also foreshadows a bright future which we see as a further deepening of our understanding of the 'why' and the 'how' of all of this, driven forward through our gaining a deeper understanding of the structure of the nucleons themselves - and their interactions with one another - that are primary forcing functions within the universe in which we all live, and in so doing, a further narrowing of the gap that remains to be filled in efforts to build a bridge of understanding between low-energy and high-energy nuclear physics. A unified view of nuclear physics should be next-up, albeit perhaps awaiting the collective genius of yet another generation of student physicists!

\section{Acknowledgment}

Authors acknowledge the friendship and support of many collaborators within the US and abroad who contributed directly and indirectly to the development of the SA-NCSM, especially through the sharing of many well-prepared students who enabled our program to grow and expand over the years. Special thanks for technical support from David Kekejian in the preparation this manuscript, and for allowing use of some key (unpublished) concepts from his thesis.

This work was supported in part by the U.S. National Science Foundation (PHY1913728) and the Czech Science Foundation (16-16772S). It benefited from high performance computational resources provided by LSU (www.hpc.lsu.edu), the National Energy Research Scientific Computing Center (NERSC), a U.S. Department of Energy Office of Science User Facility operated under Contract No. DE-AC02-05CH11231, as well as the Frontera computing project at the Texas Advanced Computing Center, made possible by National Science Foundation award OAC-1818253.

\section{References}

[1] E. Rutherford, H. Geiger (1908) The charge and nature of the alpha-particle. Proceedings of the Royal Society of London. Series A, Containing Papers of a Mathematical and Physical Character 81(546) 162-173.

[2] N. Bohr (1913) I. On the constitution of atoms and molecules. The London, Edinburgh, and Dublin Philosophical Magazine and Journal of Science 26(151) 1-25. 


\section{J.P. Draayer, D. Kekejian, G.H. Sargsyan, T. Dytrych, K.D. Launey}

[3] A. Bohr, B.R. Mottelson (1975) "Nuclear Structure, Vol. II: Nuclear Deformation". W.A. Benjamin, Reading, Massachusetts, USA, 1975.

[4] M.G. Mayer, J.H.D. Jensen (1955) "Elementary theory of nuclear shell structure". Wiley.

[5] P.O. Hess, M. Seiwert, J. Maruhn, W. Greiner (1980) General collective model and its application to ${ }_{92}^{238} \mathrm{U}$. Zeitschrift fuer Physik A, Atoms and Nuclei 296 147-163.

[6] J.P. Elliott (1958) Collective motion in the nuclear shell model. I. Classification schemes for states of mixed configurations. Proc. R. Soc. Lond. A 245 128-145.

[7] F. Iachello, A. Arima (1987) The Interacting Boson Model. Cambridge University Press, Cambridge, UK.

[8] T. Dytrych, K.D. Sviratcheva, C. Bahri, J.P. Draayer, J.P. Vary (2007) Evidence for symplectic symmetry in ab initio no-core shell model results for light nuclei. Phys. Rev. Lett. 98162503.

[9] T. Dytrych, K.D. Launey, J.P. Draayer, P. Maris, J.P. Vary, E. Saule, U. Catalyurek, M. Sosonkina, D. Langr, M.A. Caprio (2013) Collective modes in light nuclei from first principles. Phys. Rev. Lett. 111252501.

[10] T. Dytrych, K.D. Launey, J.P. Draayer, D.J. Rowe, J.L. Wood, G. Rosensteel, C. Bahri, D. Langr, R.B. Baker (2020) Physics of nuclei: Key role of an emergent symmetry. Phys. Rev. Lett. 124042501.

[11] K.D. Launey, T. Dytrych, J.P. Draayer (2016) Symmetry-guided large-scale shellmodel theory. Progress in Particle and Nuclear Physics 89 101-136.

[12] P. Navrátil, J.P. Vary, B.R. Barrett (2000) Properties of ${ }^{12} \mathrm{C}$ in the ab initio nuclear shell model. Phys. Rev. Lett. 84 5728-5731.

[13] G. Rosensteel, J.P. Draayer, K.J. Weeks (1984) Symplectic shell-model calculation for ${ }^{24} \mathrm{Mg}$. Nucl. Phys. A 419(1) 1-12.

[14] J.P. Draayer, K.J. Weeks, G. Rosensteel (1984) Symplectic shell-model calculations for ${ }^{20} \mathrm{Ne}$ with horizontal configuration mixing. Nucl. Phys. A 413(2) 215-222.

[15] O. Castanos, J.P. Draayer (1989) Contracted symplectic model with ds-shell applications. Nucl. Phys. A 491(3) 349-372.

[16] O. Castanos, P.O. Hess, J.P. Draayer, P. Rochford (1991) Pseudo-symplectic model for strongly deformed heavy nuclei. Nucl. Phys. A 524(3) 469-478.

[17] G. Rosensteel, D.J. Rowe (1977) Nuclear Sp(3, R) model. Phys. Rev. Lett. 38 1014.

[18] A.C. Dreyfuss, K.D. Launey, T. Dytrych, J.P. Draayer, C. Bahri (2013) Hoyle state and rotational features in carbon-12 within a no-core shell-model framework. Phys. Lett. B 727(4) 511-515.

[19] G.K. Tobin, M.C. Ferriss, K.D. Launey, T. Dytrych, J.P. Draayer, A.C. Dreyfuss, C. Bahri (2014) Symplectic no-core shell-model approach to intermediate-mass nuclei. Phys. Rev. C 89034312.

[20] K.D. Launey, A. Mercenne, T. Dytrych (2021) Nuclear dynamics and reactions in the ab initio symmetry-adapted framework. Annu. Rev. Nucl. Part. Sci. 71253.

[21] G. Sargsyan (2021) Electromagnetic transitions and beta decays in nuclei from the ab initio symmetry-adapted no-core shell model. LSU Doctoral Dissertations 5641.

[22] D. Kekejian. Symplectic effective field theory. To be submitted. 